\title{
1 Repeated global migrations on different plant hosts by the tropical pathogen
}

2 Phytophthora palmivora

4 Jianan Wang ${ }^{1}$, Michael D. Coffey ${ }^{2}$, Nicola De Maio ${ }^{3,4}$, Erica M. Goss ${ }^{1 *}$

$6{ }^{1}$ Department of Plant Pathology and Emerging Pathogens Institute, University of Florida,

7 Gainesville, FL, USA 32611;

$8{ }^{2}$ Department of Plant Pathology and Microbiology, University of California, Riverside, CA,

9 USA 92521;

$10{ }^{3}$ Nuffield Department of Medicine, University of Oxford, OX3 7BN, Oxford, United Kingdom.

$11{ }^{4}$ European Molecular Biology Laboratory, European Bioinformatics Institute (EMBL-EBI),

12 Wellcome Genome Campus, Hinxton CB10 1SD, UK

13

$14 \quad$ *e-mail: emgoss@ufl.edu

16 Keywords: host tracking, host shift, Oomycete, genetic diversity, structured coalescent analysis

18 Running title: Host tracking by a broad host range plant pathogen

19 ORCID ID:

20 Jianan Wang: https://orcid.org/0000-0002-7704-666X

21 Nicola De Maio: https://orcid.org/0000-0002-1776-8564

22 Erica M. Goss: https://orcid.org/0000-0003-3512-2107 


\section{Abstract}

26 The genetic structure and diversity of plant pathogen populations are the outcomes of

27 evolutionary interactions with hosts and local environments, and migration at different scales,

28 including human-enabled long-distance dispersal events. As a result, patterns of genetic variation

29 in present populations may elucidate the history of pathogens. Phytophthora palmivora is a

30 devastating oomycete that causes disease in a broad range of plant hosts in the tropics and

31 subtropics worldwide. The center of diversity of P. palmivora is in Southeast Asia, but it is a

32 destructive pathogen of hosts native to South America. Our objective was to use multilocus

33 sequence analysis to resolve the origin and historical migration pathways of $P$. palmivora. Our

34 analysis supports Southeast Asia as a center of diversity of $P$. palmivora and indicates that a

35 single colonization event was responsible for the global pandemic of black pod disease of cacao.

36 Analysis using the structured coalescent indicated that $P$. palmivora emerged on cacao and that

37 cacao has been the major source of migrants to populations in Asia, Africa, and Pacific Islands.

38 To explain these results, we hypothesize widespread introgression between the pandemic cacao

39 lineage and populations native to Asia and the Pacific Islands. The complex evolutionary history

40 of $P$. palmivora is a consequence of geographic isolation followed by long-distance movement

41 and host jumps that allowed global expansion with cacao, coconut and other hosts. 
45 With continued growth of human populations and change in global climate, there is increasing

46 concern over plant diseases affecting food crop and economic security ${ }^{1}$. Epidemics of plant

47 disease can result in major yield losses and associated economic consequences ${ }^{2}$. In low- and

48 middle-income countries, plant diseases can have a relatively larger impact on local socio-

49 economic development ${ }^{3,4}$. Understanding the population biology and evolution of plant

50 pathogens can aid in disease management. For example, determining the geographic history of a

51 plant pathogen can help locate plant germplasm that co-evolved with the pathogen and exhibits

52 disease resistance, which can be employed in plant breeding programs. Knowledge of plant

53 pathogen migration patterns can lead to informed decision-making for disease prevention,

54 specifically to limit re-introductions and pathogen re-emergence ${ }^{5}$. Global pandemics may

55 originate from a very successful invasive population that itself is responsible for multiple

56 secondary invasions $\mathrm{s}^{3,6-8}$. Hence, mitigation strategies may specifically target invasive

57 populations or genotypes ${ }^{9,10}$.

However, the above efforts can be hampered by pathogens making dramatic host jumps.

59 Host jumps are pervasive in the emergence or spread of plant pathogens and have been identified

60 as a crucial mechanism underlying pathogen diversification and ultimately speciation ${ }^{11,12}$.

61 International travel and global trade over the course of human history have exposed hosts to new

62 pathogens, thus, facilitating host jumps ${ }^{1,3,9,13-15}$. Deciphering complex interactions among plant

63 hosts, pathogens and human activities can elucidate major drivers behind the emergence, re-

64 emergence and dispersal of plant pathogens ${ }^{1}$. Many such studies of pathogen emergence have

65 targeted major crop pathogens that exhibit relatively narrow host associations ${ }^{5,16-21}$. When

66 pathogens have multiple host associations, can the influence of different hosts on pathogen

67 emergence and dispersal be resolved? 
Phytophthora is a genus of plant-damaging Oomycetes, including more than 100 described species ${ }^{22-25}$. Phytophthora species can infect a broad range of plant hosts ${ }^{26}$ and have caused enormous economic losses to agro-ecosystems and ecological damage in natural ecosystems. The relatively narrow host range pathogen Phytophthora infestans, causal agent of

72 potato and tomato late blight, was globally distributed on potato, leading to the Great Irish

73 Famine. In 2008, P. infestans spread across the Eastern United States on tomato plants for home

74 gardens $^{27}$. P. ramorum is a broad host range pathogen, which dispersed on ornamental plants

75 followed by host jumps to timber and forest $\operatorname{trees}^{28}$. The resulting disease outbreaks in North

76 America and Europe have been economically costly and have changed the ecology of coastal

77 California forests ${ }^{29}$. Phytophthora palmivora (Butler) Butler (1919) is a destructive tropical and

78 subtropical plant pathogen with a very broad host range in the tropics and subtropics ${ }^{30,31}$. $P$.

79 palmivora causes problematic diseases of coconut and other palms (bud rot), cacao (black pod,

80 canker, and cherelle wilt), rubber (black stripe), durian (fruit rot and canker), orchids and other

81 ornamentals, and more ${ }^{26}$. Annual losses due to black pod of cacao, caused primarily by $P$.

82 palmivora, have been estimated at more than US\$400 million per year ${ }^{103}$ and bud rot endemics

83 have affected more than 70,000 ha of oil palm in Colombia ${ }^{32}$. However, like many other tropical

84 pathogens, research on $P$. palmivora population biology and genetics has lagged far behind

85 temperate Phytophthora, even though this pathogen is responsible for significant economic

86 losses. To advance research on P. palmivora, the genome of an isolate from cacao was recently

87 sequenced. Estimated genome size was greater than 151.23 Mb with 44327 genes and initial

88 analysis of gene models indicated that P. palmivora experienced a genome doubling event ${ }^{33}$.

89 The hypothesized center of origin of $P$. palmivora has changed over time. Initially,

90 Central or South America was suspected to be the native region of the pathogen, because of the 
91 susceptibility of indigenous hosts and the apparent global distribution of the pathogen on cacao

92 (Theobroma cacao), which is native to South America ${ }^{34}$. Findings of high levels of genetic

93 diversity in P. palmivora populations in Southeast Asia, particularly among isolates from native

94 hosts coconut and durian, led to the proposal that Southeast Asia is the center of origin of $P$.

95 palmivora ${ }^{30,35}$. Recent advances in population genetics and coalescent model-based approaches

96 have revolutionized methods to identify plant pathogen centers of origin, reconstruct migration

97 pathways and reveal population genetic structure ${ }^{36-44}$. These new tools have substantially

98 advanced knowledge of the evolutionary history and epidemiology of major plant pathogens, but

99 have not been applied towards understanding the global spread of P. palmivora.

The main objective of this study was to describe the global population structure of $P$.

101 palmivora and historical migration pathways using Bayesian and coalescent model-based

102 inference approaches. We specifically addressed the following questions: (1) Are populations of

103 P. palmivora structured by host or geography? (2) Where is the center of origin of P. palmivora?

104 (3) What were the main migration pathways out of the center of origin? (4) Did host shifts drive

105 the global expansion of the P. palmivora?

106

\section{Results}

108 Nucleotide diversity by geographic region. We evaluated DNA sequence variation in four

109 genes (one mitochondrial and three nuclear) for three major geographic regions (Table 1). Higher

110 values of average pairwise nucleotide diversity $(\pi)$ and Watterson's theta $(\theta \mathrm{w})$ were observed in

111 the Asia-Pacific region than in Africa and the Americas. Tajima's D and Fu \& Li's D* and F*

112 test statistics were not significantly different from zero except for the sample from the Americas

113 (Table 1). A significantly positive value of Tajima's D was observed for trpl in the Americas, 
114 indicating more intermediate frequency alleles than expected under neutral evolution, while all

115 three test statistics were significantly negative for coxII in the Americas, indicating excessive

116 rare polymorphisms.

117 Population subdivision and structure. Population differentiation was tested by AMOVA

118 (Table 2). All four loci showed a significant portion of genetic variation (8-12\%) distributed

119 among regions (Africa, Americas and Asia-Pacific). Differentiation between the two most

120 common hosts in the data set, coconut and cacao, explained around one quarter of the genetic

121 variation in the nuclear loci (23-27\%).

122 To further explore population structure, $P$. palmivora isolates were grouped by Bayesian

123 clustering, using an allelic data set from the three nuclear loci (Fig. 1). When the number of

124 clusters (K) was set to 2, most isolates from the Asia-Pacific region were in one cluster, and the

125 majority of isolates from Africa and Americas were in the other. As $\mathrm{K}$ was increased, isolates

126 from Indonesia, Philippines, Malaysia, and Pacific Island formed new clusters. Based on delta K,

127 the optimum value of $\mathrm{K}$ was 2 . The genetic structure within the Asia-Pacific region was greater

128 than in Africa and the Americas. The small sample from south Asia (India and Sri Lanka)

129 resembled populations in Africa and the Americas, the majority of which were isolated from

130 cacao. Bayesian clustering of clone-corrected data produced the same overall pattern as the full

131 data set (Supplementary Fig. 1).

132 The non-parameterized clustering method DAPC showed the first discriminant

133 component separating isolates collected from cacao in all geographic regions from isolates

134 collected from coconut and other hosts (Fig. 2). The second discriminant component separated

135 isolates sampled from durian and other hosts from coconut and cacao isolates. Six isolates 
136 collected from coconut and other hosts in the Asia-Pacific and Americas clustered with isolates

137 from cacao, consistent with isolates shifting from cacao to other hosts.
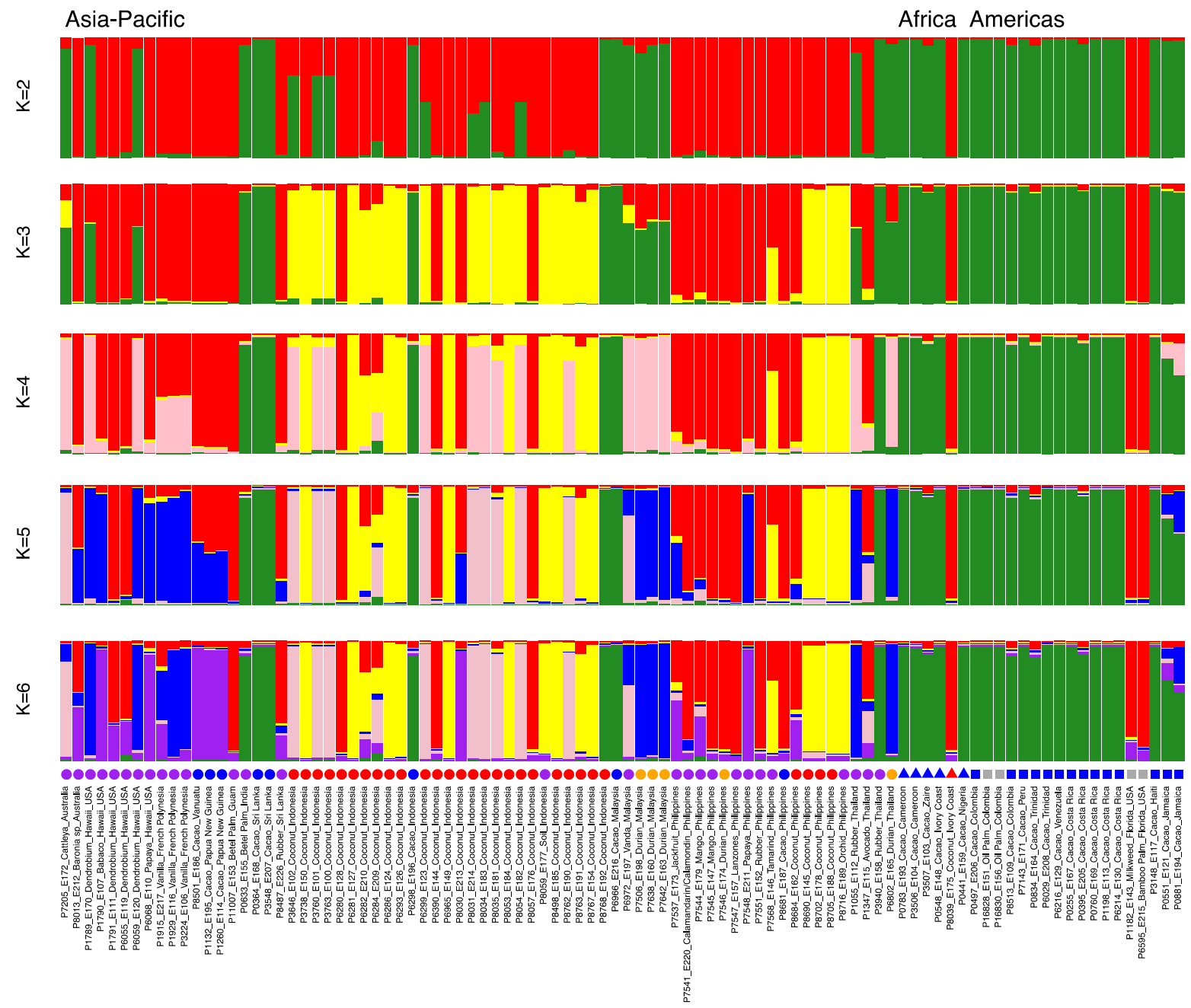

139 Fig. 1. STRUCTURE assignments of $\boldsymbol{P}$. palmivora isolates to populations for values of $\mathbf{K}$

140 from 2 to 6. Greater genetic structure is apparent in the Asia-Pacific region. Origins of isolates

141 are indicated below each bar. Isolates from cacao are indicated by a blue symbol, those from

142 coconut with a red symbol, and those from durian with an orange symbol. Isolates with purple or

143 gray symbols are from other hosts. Region is indicated by the shape of the symbol (circle for

144 Asia-Pacific, triangle for Africa, square for Americans). 


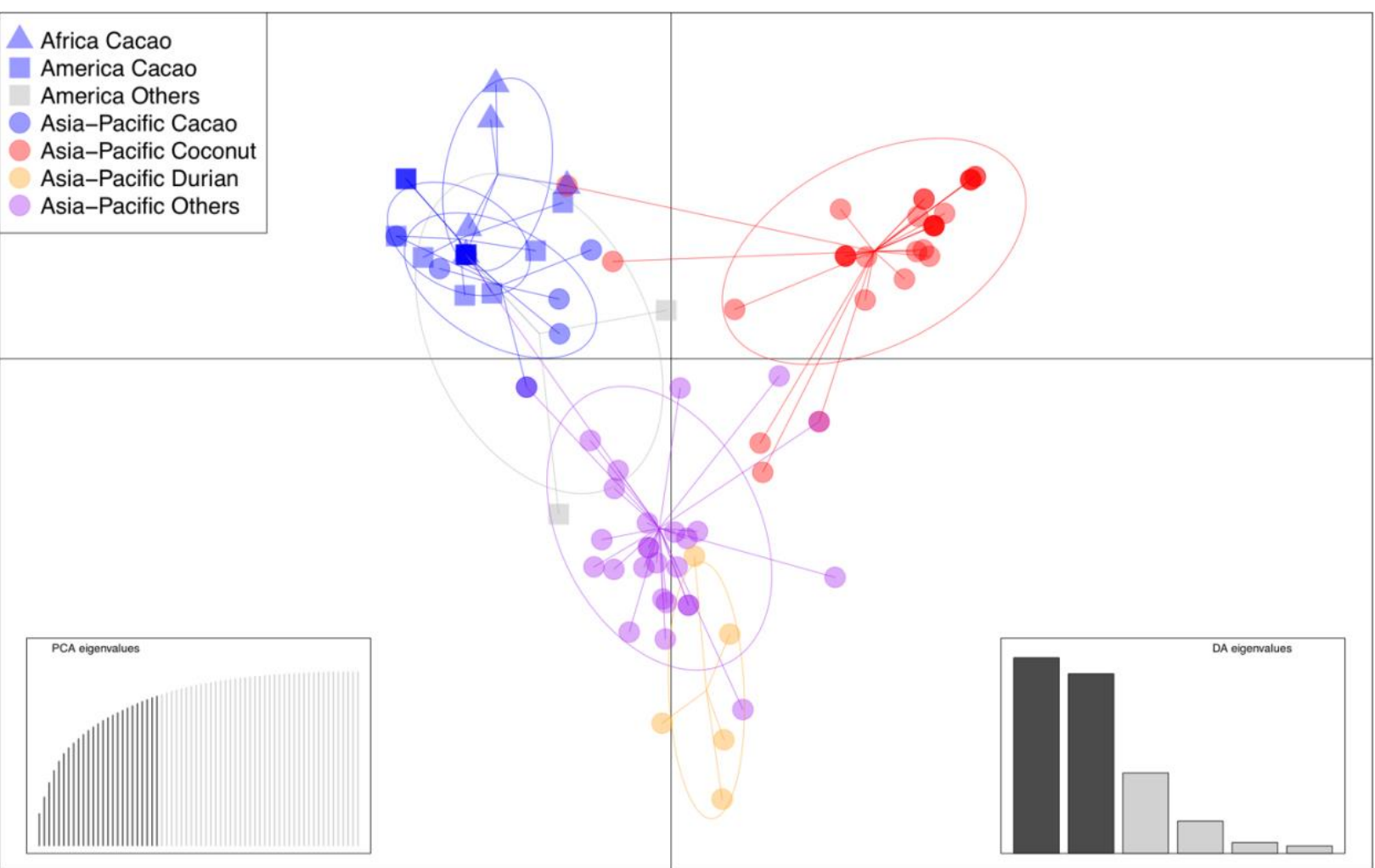

Fig. 2. DAPC clusters of $\boldsymbol{P}$. palmivora isolates from different hosts and geographic regions.

147 All isolates collected from cacao in the three geographic regions grouped together. Asia-Pacific

148 isolates sampled from coconut and durian grouped into two largely distinct clusters.

150 Global migration patterns. Discrete Bayesian phylogeographic analysis was used to infer the

151 geographic location of the root state of each locus ${ }^{45,46}$. The reconstructed root states supported

152 Indonesia and/or Philippines as the inferred evolutionary origin of our sample (Fig. 3). The

153 posterior probabilities for Philippines or Indonesia as the root state varied by locus

154 (Supplementary Table 4). The corresponding analysis with BASTA, which is not as biased by

155 sampling patterns, produced similar maximum clade credibility genealogies but with low

156 posterior probabilities for geographic root states. 

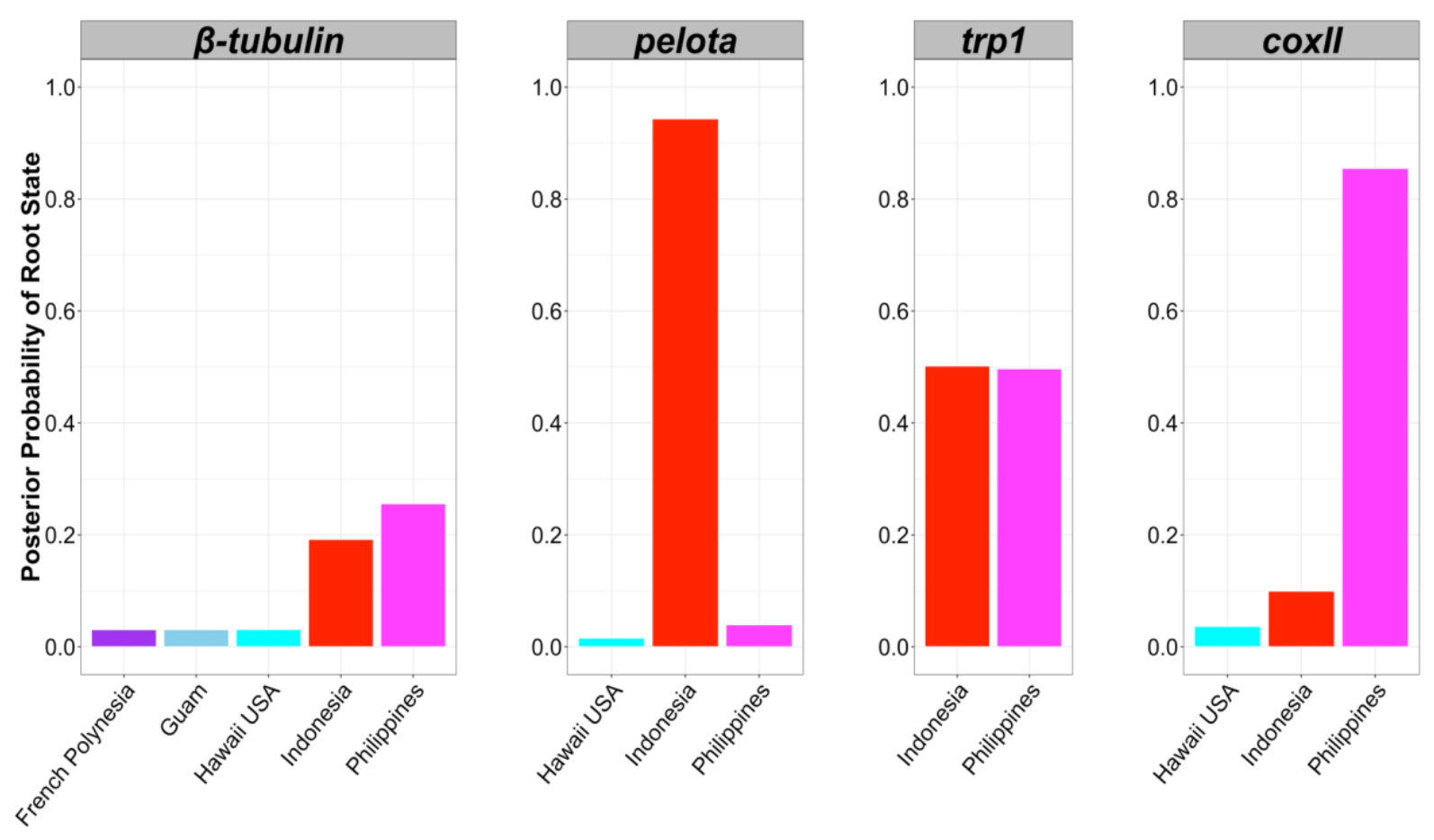

Fig. 3. Root state posterior probability values inferred using discrete phylogeographic analysis for each location (country) by locus: $\beta$-tubulin, pelota, trp1 and coxII. The posterior probabilities for an Indonesia root (red bar) and/or a Philippines root (magenta bar) are higher than for the other locations (other colors) for each locus, inferred independently. Due to limited space, only five locations with the five highest posterior probabilities for $\beta$-tubulin are shown.

163 For pelota, trpl and cox II, only the locations with posterior probabilities more than 0.01 are 164 shown.

167 2-4) indicate that lineages presently associated with Southeast Asia and the Pacific Islands

168 diverged much earlier than lineages associated with Central and South America and Central

169 Africa (mainly isolated from cacao). The mitochondrial locus, coxII, produced three lineages,

170 one of which shows early diversification and is represented by isolates from SE Asia, Australia,

171 Papua New Guinea, Vanuatu, French Polynesia, Hawaii, Ivory Coast and Jamaica (Fig. 4). 
172 Across loci, isolates from Pacific Islands represented lineages that diverged prior to

173 diversification of cacao-associated lineages. We observed that isolates from different hosts

174 within given geographic regions had distinct evolutionary histories. For example, an isolate

175 collected from coconut in Ivory Coast had distinct ancestry from the cacao isolates collected in

176 Central Africa, and this was also observed for isolates from Florida and Jamaica. For the nuclear

177 loci, we observed that most of the isolates from cacao exhibited two distinct haplotypes while

178 showing little variation within each haplotype among isolates, consistent with a clonal lineage.

179 Isolates from Indonesia and Philippines were less likely to have two diverged alleles but they

180 exhibited more variation among isolates.

\begin{tabular}{|l}
\hline Locations \\
\hline India \\
Sri Lanka \\
Thailand \\
Malaysia \\
Philippines \\
Indonesia \\
Australia \\
Guam \\
Papua New Guinea \\
Vanuatu \\
French Polynesia \\
Hawaii USA \\
Cameroon \\
Zaire \\
Ivory Coast \\
Nigeria \\
Colombia \\
Peru \\
Trinidad \\
Venezuela \\
Costa Rica \\
Florida USA \\
Jamaica \\
Haiti
\end{tabular}

182 Fig. 4. Maximum clade credibility genealogy for the coxII locus. Colors of branches indicate 183 the most probable geographic origin of each lineage.

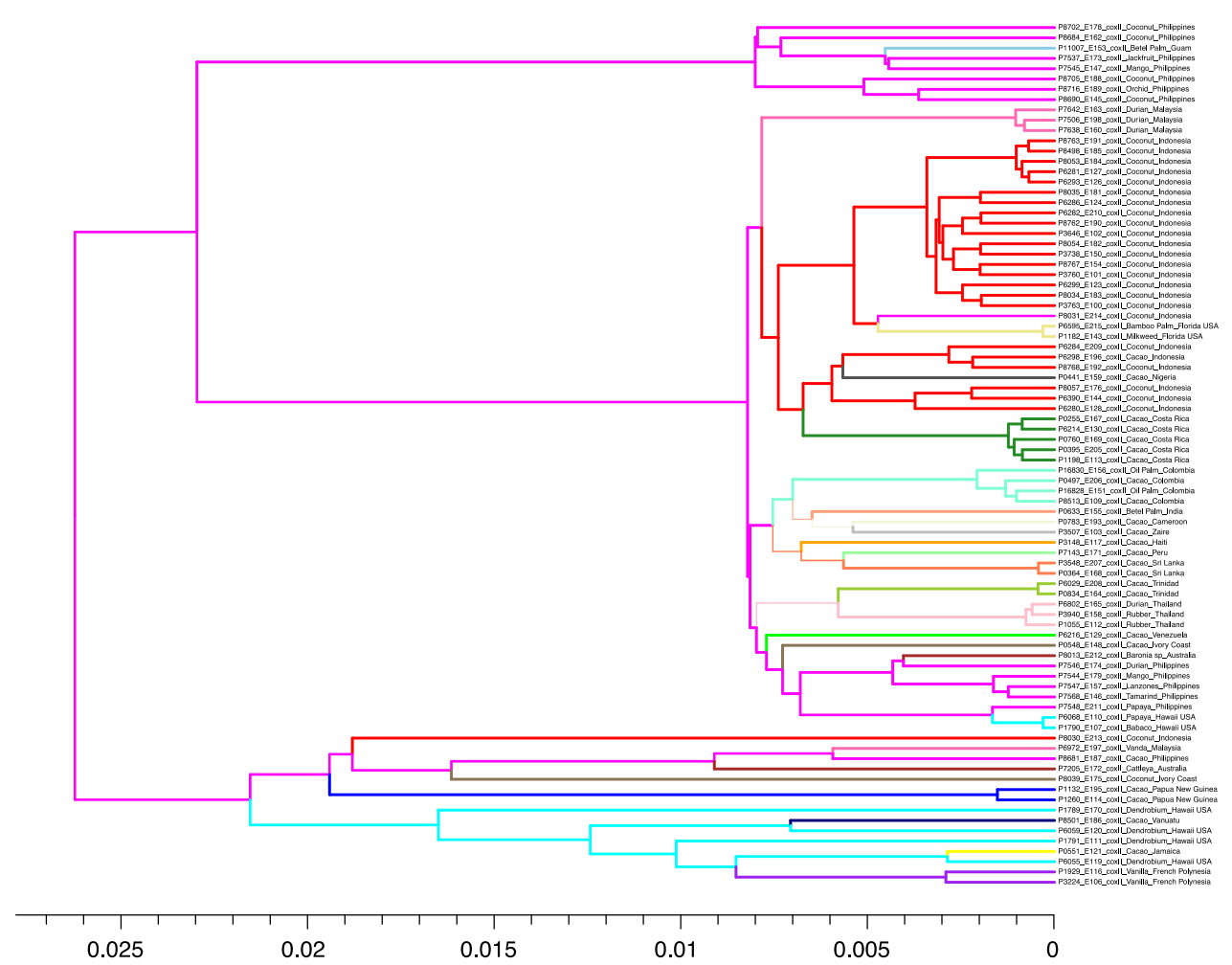


185 Host switching. To complement the phylogeographic analysis and infer patterns of historical

186 migration between major host groups, we conducted a multi-locus BASTA analysis by host

187 group and region (Americas, Asia-Pacific and Africa). Based on analyses of genetic structure

188 and phylogeography, we expected that migration occurred from coconut or other native hosts in

189 SE Asia to hosts in the Pacific, to cacao in the Americas, and then back to cacao and other hosts

190 in Asia. The BASTA analysis did not confirm this pattern. Using a two-host model to determine

191 the directionality of gene flow between cacao and coconut, we inferred cacao to be the root host

192 with high posterior probabilities ( $\geq 0.96$ for each locus) and significant migration from cacao to

193 coconut (Supplementary Table 5a). We examined if the existence of unsampled populations

194 might have affected the analyses using a three-host model with one unsampled host. Cacao and

195 the unsampled host were inferred as the root hosts with nearly even posterior probabilities. Mean

196 rates of migration from cacao to coconut and the unsampled host to coconut were moderate to

197 high, but the 95\% HPD of the migration rates from the unsampled host included zero

198 (Supplementary Table 5b), indicating uncertainty in these estimates. To incorporate geography,

199 we examined a three-deme model, with populations cacao-America, cacao-Asia-Pacific and

200 coconut-Asia. Cacao-America was inferred as the root state with high posterior probabilities

201 ( $\geq 0.93$ for each locus). Cacao-America was the major source of migrants, while cacao-Asia-

202 Pacific and coconut-Asia were the major sinks (Table 3A; Supplementary Figs. 5-8).

203 Incorporating an unsampled population into this model produced uncertainty in the root state

204 with posterior probabilities of $0.63-0.68$ for cacao-Asia-Pacific and $0.27-0.31$ for the unsampled

205 population and wide 95\% HPDs on immigration estimates from the unsampled population (Table

206 3b). To explore if all remaining hosts, other than coconuts and cacao, in the Asia-Pacific region

207 could represent the unsampled population, we examined a four-deme model replacing the 
unsampled deme with a putative deme we designated Other-Asia-Pacific. Similar to the previous models without an unsampled population, cacao-America was inferred as the root state with high posterior probabilities ( $\geq 0.96$ for each locus), and cacao-Asia-Pacific and coconut-Asia were the

211 two major sinks. Other-Asia-Pacific was inferred as a third major sink (Table 3c), which is the

212 opposite migration direction to the models incorporating an unsampled deme. Models that

213 explicitly incorporated the few cacao isolates from Africa produced results generally consistent

214 with the models without the cacao-Africa deme (Supplementary Table 6a, b).

216 Discussion

217 The evolutionary history of crop pathogens is as complex as the phylogeographic and

218 agricultural histories of their hosts and the human populations that have cultivated them. Our

219 multilocus sequence analysis of a global sample of Phytophthora palmivora isolates revealed

220 population subdivision among three major geographic regions (Africa, Americas, Asia-Pacific)

221 and between hosts, here highly sampled cacao and coconut. We found greater genetic diversity

222 and more complex population structure within Southeast Asia as compared to other global

223 regions, and reduced genetic variation among isolates collected from cacao. Therefore, our

224 results generally support Southeast Asia as the P. palmivora center of diversity ${ }^{30,35}$. On the other

225 hand, our analyses suggest that the Americas are a significant source of the genetic diversity now

226 observed in the Asia-Pacific region. Here, we argue that the complex evolutionary history of $P$.

227 palmivora reflects the history of human travel and trade in the tropics.

229 island chains, which naturally introduce geographic isolation due to historically infrequent

230 movement of plant hosts, including by human migrations ${ }^{47}$. Genetically diverse isolates were 
231 associated with coconut palms and a variety of other tropical hosts. However, because the

232 distribution of hosts sampled was not independent of geographic region, we cannot fully separate

233 the relative influence of host and geography. For example, American and African isolates largely

234 clustered together and separately from isolates from the Asia-Pacific region, but cacao was the

235 most-sampled host in Africa and the Americas. Indeed, the population genetic structure of $P$.

236 palmivora on cacao suggests that a single colonization was responsible for the global pandemic

237 of $P$. palmivora causing black pod of cacao. The globally successful "cacao strain" is

238 characterized by a single coxII haplotype and STRUCTURE cluster based on the genotypes of

239 three nuclear loci. The most frequent haplotypes for each locus are associated with cacao. Many

240 of the isolates from the Americas, Africa, and South Asia represented this cacao-associated

241 lineage. The resulting pattern of genetic variation in the Americas produced positive values of

242 Tajima's D test across nuclear housekeeping genes and a statistically significant negative value

243 for the mitochondrial coxII locus. These values are consistent with a recent population

244 bottleneck $^{48}$. In the Americas and South Asia, the "cacao strain" was isolated from palms and

245 rubber, suggesting movement from cacao to other hosts. For example, two isolates obtained from

246 cacao and oil palm in Colombia had identical sequences at the four sequenced loci. These

247 isolates shared haplotypes with isolates from cacao worldwide and commercial oil palm

248 production only began in Colombia in $1945^{102}$, therefore we infer that $P$. palmivora colonized oil

249 palm from cacao.

250 We conducted phylogeographic analyses to infer the potential historical processes behind

251 the observed patterns of genetic variation. Using discrete phylogeography, we inferred root states

252 of our P. palmivora sample to be Indonesia or the Philippines, depending on the locus. We might

253 expect that the pathogen has a metapopulation structure across these island nations, mediated by 
sea level changes joining or separating islands, dispersal of infected fruits following sea currents,

255 or, more recently, trade in hosts infected by $P$. palmivora. The corresponding BASTA analysis

256 was not able to infer a root location, and our reduced parameter analyses that grouped isolates by

257 geographic region and host group inferred an American origin. BASTA is less susceptible to the

258 effects of sampling bias, but inference of an American origin was unexpected because of the

259 higher genetic diversity of the pathogen in the Asia-Pacific region. Simulations suggest that high

260 posterior probabilities provided by the discrete model likely underestimate posterior

261 uncertainty ${ }^{44}$. Our discrete model results reflect deep sampling in Southeast Asia, while the

262 BASTA results indicate that sampling across a greater diversity of hosts and locations is needed.

263 A long history of controversy regarding the geographic and evolutionary origin of the late blight

264 pathogen, $P$. infestans, has been exacerbated by limited sampling of wild relatives of potato in

265 the South American Andes ${ }^{49,50}$ and the geographic origins of many other widespread

266 Phytophthora pathogens are unknown.

Migration estimates over the history of our sample of $P$. palmivora, inferred by BASTA,

268 suggests movement from cacao in the Americas to cacao in Asia and Africa, and from cacao to

269 other hosts in Asia. Because we observed genetic diversity on other hosts, it is unlikely that the

270 sole origin of the pathogen is cacao. Indeed, we could not exclude the presence of an alternative,

271 unsampled source population. In our inferred genealogies, we observed that haplotypes were not

272 segregating independently, particularly for isolates from cacao, which likely represent an

273 asexually reproducing lineage. The phylogeny for the coxII mitochondrial locus showed two

274 groups of haplotypes, a large group of closely related haplotypes and a second group of low

275 frequency, diversified haplotypes, which could represent different histories of ancestral

276 populations of $P$. palmivora. One explanation for the observed patterns is introgression, perhaps 
277 repeated introgression, from the pandemic lineage on cacao to endemic populations of

278 Phytophthora in Southeast Asia and the Pacific Islands. We do not yet have evidence of

279 populations of $P$. palmivora or a closely related species on wild plants in the Americas which

280 could have sourced migration to Asia. However, P. palmivora infects important tropical cash

281 crops native to South America, including cacao ${ }^{52}$, ubber $^{53}$, and neotropical orchids ${ }^{54}$. Genome

282 sequencing of an isolate of $P$. palmivora from cacao indicates that this pathogen underwent

283 genome doubling. We speculate that genome doubling was the consequence of a hybridization

284 event between strains of different geographic origin, because genome doubling is commonly

285 associated with interspecific hybridization ${ }^{51}$.

We can draw parallels between global patterns in the genetic variation of $P$. palmivora

287 and historical global movement of the hosts considered here (Fig. 5). The Philippines represents

288 one of two independent domestications of coconut (Cocos nucifera $)^{47,55}$. Historical and genetic

289 data have clarified the routes of coconut as it spread throughout the tropics and subtropics ${ }^{55}$.

290 Various lines of evidence suggest that Pacific coconut was brought from the Philippines to

291 eastern Polynesia and to the Pacific coast of Latin America by pre-Columbian Austronesians.

292 Trade occurred in the opposite direction as well, because herbarium specimens and

293 anthropological studies suggest pre-historic introduction of sweet potato from the Pacific coast of

294 South America to Polynesia ${ }^{56}$. Later, the Portuguese set up plantations of the South Asian

295 domestication (Indo-Atlantic genotypes) of coconut in West Africa, Brazil, and the Caribbean.

296 Our findings of early divergence in our $P$. palmivora sample from the Pacific Islands is

297 consistent with early movement, possibly on Pacific coconut, whereas isolates from Ivory Coast

298 and Jamaica with deep nodes suggest movement on Indo-Atlantic coconut. $P$. palmivora infects

299 numerous other tropical fruits and ornamentals native to the Asia-Pacific region, on which the 
A. Historical movement of major hosts

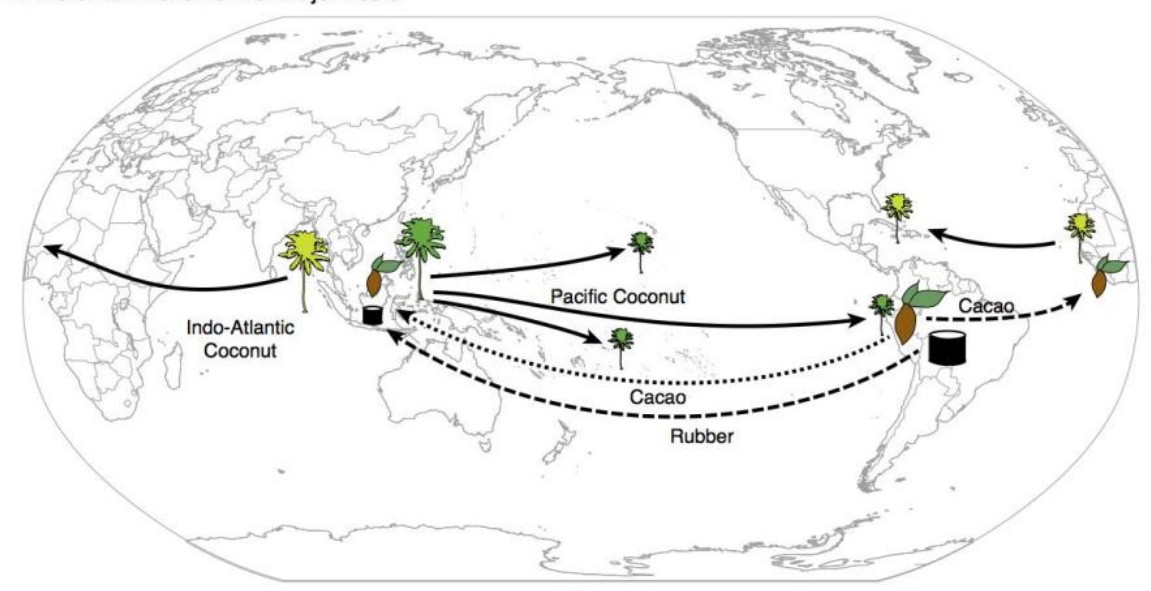

B. Inferred P. palmivora migration rates between regions and host groups

Fig. 5. Movement of major hosts of $P$. palmivora out of their centers of origin and inferred palmivora hosts. Indo-Atlantic and Pacific coconut were domesticated in South and Southeast Asia, respectively. Cacao and rubber originated in South America. Many other tropical crops host $P$. palmivora, including ornamentals. (b) Summary of BASTA inferences of P. palmivora migration (solid lines, width proportional to mean migration rate) and potential migration from an unsampled deme (dotted lines). BASTA inferred high migration rates from cacao in the Americas to cacao in Asia-Pacific and Africa, and migration from cacao in Asia-Pacific to coconut and other hosts (indicated by a durian fruit), as well as migration from cacao in the Americas directly to other Asia-Pacific hosts. 
pathogen could have dispersed or persisted over hundreds of years. If we assume that the

313 divergence in sequences of isolates from Hawaii and other Pacific Islands are associated with

314 human colonization of the Eastern Pacific islands around 1200 AD, we find that the colonization

315 of global cacao production is during the last 150-200 years, as expected. The major documented

316 movements of cacao to Africa and Asia was during the colonial period, and originated from

317 Venezuela, Trinidad and Brazil ${ }^{52,57}$. Rubber is another major South American export and host of

318 P. palmivora, with modern plantations located in South and Southeast Asia and West Africa ${ }^{59,60}$.

319 The history of rubber in Asia started with the introduction of specimen trees from the Amazon

320 region of Brazil to Ceylon, Singapore, and Penang in 1876, and rubber was established in

321 plantations in Malaysia through $1898^{58}$.

322 We hypothesize that the diversity and genetic divergence of $P$. palmivora isolates in the

323 Asia-Pacific region is an example of host-tracking. Our suggest that there may a long history of

324 association of $P$. palmivora with coconut. Alternatively, $P$. palmivora may have long-term

325 associations with other hosts native to Southeast Asia and coconut served primarily as an early

326 vector for long-distance dispersal. The host-tracking model of plant pathogen emergence has

327 been seen in other host-pathogen systems in Southeast Asia, such as the black Sigatoka pathogen

328 Mycosphaerella fijiensis on banana ${ }^{61}$ and blast fungus Magnaporthe oryzae on rice ${ }^{62}$. In contrast,

329 the nearly monomorphic population P. palmivora found in Americas, South Asia, and Africa can

330 be explained by a pandemic lineage of $P$. palmivora on cacao, following a genetic bottleneck

331 likely associated with initial colonization of this host. The population of $P$. palmivora associated

332 with cacao resembles the pattern of emergence of globally-distributed clonal lineages of $P$.

333 infestans on potato ${ }^{21,63-65}$. Several other oomycetes have exhibited similar genetic bottlenecks 
334 upon intercontintental spread, including Phytophthora ramorum ${ }^{28}$. These events are often

335 associated with a host jump, particularly colonization of plants for export.

336 The evolutionary and geographic history of $P$. palmivora could be clarified by

337 examination of additional isolates from uncultivated plants, which may represent older or more

338 isolated populations, or by using preserved DNA from herbarium samples. In general, the

339 distributions of crop pests and pathogens are highly dependent on the distribution of their hosts,

340 even though the specific mechanisms of global spread differ among species ${ }^{66}$. The idiosyncrasies

341 of plant movement during human history shaped the genetic variation and structure of modern

342 populations of cultivated plants and of their pathogens. Therefore, efforts to manage disease may

343 benefit from teasing apart historical dependencies that structure host and pathogen genetic

344 variation and that are likely to mediate the plant-pathogen interaction.

346 Methods

$347 \boldsymbol{P}$. palmivora isolates. Genomic DNA was provided from 95 isolates of $P$. palmivora in the

348 World Oomycete Genetic Resource Collection at the University of California, Riverside, USA.

349 The isolates were originally collected from 23 hosts and 22 countries on five continents (Africa,

350 Asia, Australia, North America and South America) (Supplementary Table 1). For analysis,

351 isolates were assigned to one of three large geographical regions: 1) Asia-Pacific (Asia, Australia

352 and Pacific Islands); 2) Americas (including the Caribbean islands); and 3) Africa.

353 Multilocus sequence typing. Four housekeeping genes known to contain variation within

354 Phytophthora species were used in this study: mitochondrial gene coxII with the adjacent

355 spacer $^{67}$, and three nuclear genes, $\beta$-tubulin, pelota and trp $1^{68,69}$. Primers for the nuclear genes 
were modified for $P$. palmivora based on draft whole genome sequence kindly provided by S.

357

Schornack and S. Kamoun (Supplementary Table 2).

For PCR, a total reaction volume of $25.0 \mu \mathrm{l}$ was prepared including $1.0 \mathrm{ul}$ DNA template, 1X OneTaq master mix (NEB), 0.2uM primers and $3.0 \mathrm{uM} \mathrm{MgCl}_{2}$. PCR amplifications were performed using the following cycling protocol: initial denaturation at $94{ }^{\circ} \mathrm{C}$ for 3 minutes; followed by 35 cycles of $94{ }^{\circ} \mathrm{C}$ for 45 seconds, [ $\left(67{ }^{\circ} \mathrm{C}\right.$ for 45 seconds (pelota); $58{ }^{\circ} \mathrm{C}$ for 45 seconds $\left(\beta\right.$-tubulin); $60{ }^{\circ} \mathrm{C}$ for 45 seconds $(\operatorname{coxII}) ; 59^{\circ} \mathrm{C}$ for 60 seconds $\left.(\operatorname{trp} 1)\right)$ and $68^{\circ} \mathrm{C}$ for 1 minute; then a final extension step was $68^{\circ} \mathrm{C}$ for 10 minutes. PCR products were prepared for sequencing using ExoSAP-IT (USB Corporation, Cleveland, OH, USA) and directly sequenced in both directions at Interdisciplinary Center for Biotechnology Research (ICBR) at the University of Florida, USA. Reads were assembled and edited using software Geneious 6.1.8 (https://www.geneious.com). For the three nuclear loci, haplotype phase was inferred using the program PHASE ${ }^{70}$, assuming diploidy. The settings for PHASE were as follows: MR0 (the default model, which is the general model for recombination rate variation ${ }^{71}$ ); number of iterations $=10000$ for $\beta$-tubulin and trpl, 2000 for pelota; thinning interval=1; burn-in=100. For each nuclear locus, ten isolates that exhibited more than one heterozygous site were randomly selected and cloned using the pGEM-T Easy Vector System (Promega Corporation, Madison, WI, USA). Sequences were aligned with Geneious 6.1.8 ${ }^{72}$. Indels were removed for analysis. Nucleotide diversity and neutrality tests. Number of individuals (Nind), number of sequences (Nseq), number of haplotypes (Nhap), segregating sites (S), average pairwise nucleotide diversity $(\pi)^{73,74}$, Watterson's theta $\left(\theta_{\mathrm{w}}\right)^{75}$, Tajima's $\mathrm{D}^{76-79}$, number of mutations, number of singleton mutations, Fu and Li's $\mathrm{D}^{*}$ and $\mathrm{F}^{* 76,77,80-85}$ and minimum number of recombination events $(\mathrm{Rm})^{86}$ were determined for each of the four genes and by region using DnaSP v5 ${ }^{87}$. 
379 Population subdivision and structure. Population subdivision among the three major

380 geographic regions (Africa, Americas and Asia-Pacific) and between two major hosts (coconut,

381 native to Southeast Asia; and cacao, native to South America) were examined for each gene

382 locus by analysis of molecular variance (AMOVA) in Arlequin 3.5.2.1 ${ }^{88}$.

383 The population structure of P. palmivora was examined by model-based Bayesian

384 clustering carried out in STRUCTURE 2.3.4 $4^{36}$. For this analysis, allelic data were used from the

385 three nuclear loci. The program STRUCTURE estimates parameters independently in the

386 posterior probability distribution of allele frequencies. Parameters are estimated under the null

387 model of panmixia within populations, which is characterized by Hardy-Weinberg equilibrium at

388 each locus and an absence of linkage disequilibrium. The algorithm is robust to some departures

389 from these assumptions ${ }^{89-91}$. Based on allele frequencies at each locus, STRUCTURE assigns Q-

390 values (inferred ancestry) to each individual. Isolate P3444 was excluded, due to missing data for

391 pelota and trpl. To reduce the effect of asexual reproduction on STRUCTURE inferences, two

392 different input files were prepared. One used the full data set of 94 isolates and a second used a

393 clone-corrected data set of 69 isolates. Clone correction is a method to remove bias caused by

394 dominant or overrepresented genotypes generated by epidemic asexual reproduction. Here, we

395 clone-corrected globally by including one representative isolate of each multilocus genotype. We

396 applied the following workflow to analyze both input files. STRUCTURE was run using the

397 admixture model, and cluster numbers $(K)$ from $K=1$ to $K=15$ were evaluated using 500000

398 iterations after a burn-in period of 500000 iterations. To evaluate the stability of the results

399 across repeated runs, 20 independent runs were conducted. The runs for each value of $\mathrm{K}$ were

400 evaluated based on the second order rate of change of the likelihood function with respect to $\mathrm{K}^{92}$

401 using the online program Structure Harvester v.0.6.94 ${ }^{93}$. Due to stochastic effects among 
402 replicate STRUCTURE runs, assignment probabilities were compiled for multiple runs in the

403 program CLUMPP version 1.2, which simplifies the assessment of replicate data by calculating

404 medians $^{94}$. The parameters used were $\mathrm{M}=3, \mathrm{~W}=0$ and $\mathrm{S}=2$, GREEDY_OPTION=2, and

405 REPEATS $=10000$. The graphical visualization of the output was produced using $\mathrm{R}$ base function

406 'barplot' 95 .

407 Population subdivision, based on allelic data from the three nuclear genes, was also

408 examined using model-free discriminant analysis of principal components (DAPC) implemented

409 in the R package adegenet $1.4 \cdot 2^{41}$. DAPC was run to confirm the results of Bayesian clustering

410 approach. DAPC does not make biological assumptions and is less computationally intensive

411 than STRUCTURE. This method maximizes the variation between groups while minimizing

412 variation within group. First, DAPC transforms the data using principal components analysis

413 (PCA) and then discriminant analysis (DA) assigns individuals to clusters. The data

414 transformation ensures that the variables inputted to DA are uncorrelated and that their number is

415 less than that of analyzed individuals, so as to overcome the drawbacks of direct application of

416 DA. The appropriate number of principal components retained in the analysis can be determined

417 by cross-validation ${ }^{41}$. Because only one isolate, P8039, was in the pre-defined coconut-Africa

418 group and could not be used for in both training and validation sets, we removed this isolate and

419 retained 93 isolates for the DAPC analyses.

420 Genealogies and Phylogeographic Analysis. To infer the phylogeographic history of $P$.

421 palmivora, genealogies were inferred by Bayesian evolutionary analysis by sampling trees

422 (BEAST) and location states by discrete trait analysis ${ }^{37,46}$. We conducted separate analyses for

423 each locus, each of which is expected to have a different genealogy from the others. BEAST

424 executes ancestral reconstruction of discrete states, here location of collection, in a Bayesian 
425 statistical framework for evolutionary hypothesis testing using rooted, time-measured

426 phylogenies. In this analysis, locations associated with branch nodes were characterized by

427 continuous time Markov chain models, comparable to nucleotide, codon or amino acid

428 substitution models and Bayesian stochastic search variable selection (BSSVS) was used to

429 model the phylogeographic dispersion process ${ }^{45}$. This approach uses the geographic locations of

430 the samples to reconstruct the ancestral states of tree nodes and the root state. A strict molecular

431 clock model was used. The mutation rate was set as a constant 1.0, consequently the estimation

432 of branch lengths is in substitutions per site. We used jModelTest $2.1^{96}$ to obtain the best fitting

433 models of nucleotide substitution for each gene alignment. A coalescent tree prior and a prior of

434 constant population size were assumed. Three replications of independent MCMC were run for

435 each locus and the first $10 \%$ of recorded states were discarded as burn-in (parameter settings in

436 Supplementary Table 3). The program was run until effective sample size estimates of greater

437 than 200 were obtained, with good mixing and convergence in independent runs, as assessed in

438 Tracer v1.6 $6^{97}$. Maximum clade credibility (MCC) trees were summarized by TreeAnnotator

439 based on common ancestor height ${ }^{98}$ and visualized using $\mathrm{R}$ package $r B t^{99}$.

440 We complemented the above analysis with Bayesian structured coalescent approximation

$441(\mathrm{BASTA})^{44}$ in BEAST $2.0^{100}$. BASTA incorporates migration into the structured coalescent-

442 based model rather than modeling migration independently from the coalescent process as in the

443 above discrete phylogeographic analysis. BASTA phylogeographic inferences are less influenced

444 by sampling bias and variation in sampling intensity, and can include unsampled ghost demes.

445 All four loci were used in the analysis, were assumed to be unlinked, and were assigned different

446 nucleotide substitution models, as determined using jModeltest 2 for each locus. The symmetric

447 BSSVS with 24 locations (shown in Fig. 4) was used to model the phylogeographic dispersion 
448 process, which assumed a prior in which 60 migration rates are expected to be non-zero ${ }^{45}$. We

449 assumed the same population size for all demes. Two billion steps were used in the MCMC

450 chain for each run, and one tree was recorded every 200000 steps. To reduce the lengthy

451 running time and to obtain an effective sample size estimates of greater than 200 with good

452 mixing and convergence, two parallel runs with different seed numbers were implemented, and

453 the two parallel tree files for each locus were merged. The merged tree files were assessed in

454 Tracer v1.6 ${ }^{97}$. After the first $10 \%$ of recorded 'burn-in' states were discarded, MCC trees were

455 summarized by TreeAnnotator based on common ancestor height ${ }^{98}$.

456 We also used BASTA to explore major between-host transmissions in relation to

457 geography by estimating migration rates between demes defined by host and region. We started

458 with a simple model of the two major hosts, cacao and coconut, to determine which of these two

459 is more likely to be the ancestral host of $P$. palmivora. We incorporated geography by splitting

460 cacao isolates into cacao-Asia-Pacific and cacao-America demes, resulting in three demes:

461 cacao-America, cacao-Asia-Pacific and coconut-Asia. We also examined the effect of adding an

462 unsampled (ghost) deme. Because we did not include isolates collected from hosts other than

463 coconuts and cacao in the Asia-Pacific region, we grouped these isolates into a deme to

464 determine if these hosts represented the unsampled (ghost) deme. Finally, we examined the

465 effect on the above models of adding a cacao-Africa deme, although the sample size from Africa

466 is small. The four loci were assumed to be unlinked and assigned different nucleotide

467 substitution models, as above. We ran migration models that allowed for asymmetric migration

468 between demes, assuming the same population size for all demes. Three hundred million steps

469 were used in the MCMC chain for each run, and one tree was recorded every 100000 steps. 
Mixing was assessed and trees examined as above. The MCC trees were visualized by

IcyTree $^{101}$. Two replications of independent MCMC were run for each model.

473 Data availability

474 The DNA sequence data, inferred haplotypes and the analysis scripts that support the findings of

475 this study will be made available from the Dryad repository upon submission of this preprint to

476 another journal.

\section{References:}

1. Zhan, J., Thrall, P. H., Papaïx, J., Xie, L. \& Burdon, J. J. Playing on a pathogen's weakness: Using evolution to guide sustainable plant disease control strategies. Annu. Rev. Phytopathol. 53, 19-43 (2015).

2. Savary, S., Ficke, A., Aubertot, J.-N. \& Hollier, C. Crop losses due to diseases and their implications for global food production losses and food security. Food Sec. 4, 519-537 (2012).

3. Estoup, A. \& Guillemaud, T. Reconstructing routes of invasion using genetic data: why, how and so what? Molecular Ecology 19, 4113-4130 (2010).

4. Lawson Handley, L.-J. et al. Ecological genetics of invasive alien species. BioControl 56, 409-428 (2011).

5. Taole, M., Bihon, W., Wingfield, B. D., Wingfield, M. J. \& Burgess, T. I. Multiple introductions from multiple sources: invasion patterns for an important Eucalyptus leaf pathogen. Ecol Evol 5, 4210-4220 (2015).

6. Hänfling, B., Carvalho, G. R. \& Brandl, R. mt-DNA sequences and possible invasion pathways of the Chinese mitten crab. Marine Ecology Progress Series 238, 307-310

495 7. Downie, D. A. Locating the sources of an invasive pest, grape phylloxera, using a mitochondrial DNA gene genealogy. Mol. Ecol. 11, 2013-2026 (2002).

8. Floerl, O., Inglis, G. J., Dey, K. \& Smith, A. The importance of transport hubs in steppingstone invasions. Journal of Applied Ecology 46, 37-45 (2009).

9. Singh, R. P. et al. The emergence of Ug99 races of the stem rust fungus is a threat to world wheat production. Annual Review of Phytopathology 49, 465-481 (2011).

10. Cooke, D. E. L. et al. Genome analyses of an aggressive and invasive lineage of the Irish potato famine pathogen. PLOS Pathogens 8, e1002940 (2012).

503 11. Stukenbrock, E. H. Evolution, selection and isolation: a genomic view of speciation in 
fungal plant pathogens. New Phytologist 199, 895-907 (2013).

12. Restrepo, S., Tabima, J. F., Mideros, M. F., Grünwald, N. J. \& Matute, D. R. Speciation in fungal and oomycete plant pathogens. Annu Rev Phytopathol 52, 289-316 (2014).

13. Sax, D. F. \& Brown, J. H. The paradox of invasion. Global Ecology and Biogeography 9 , 363-371 (2000).

14. Tilman, D. et al. Forecasting agriculturally driven global environmental change. Science 292, 281-284 (2001).

15. Lockwood, J. L., Cassey, P. \& Blackburn, T. The role of propagule pressure in explaining species invasions. Trends Ecol. Evol. 20, 223-228 (2005).

16. Couch, B. C. et al. Origins of host-specific populations of the blast pathogen Magnaporthe oryzae in crop domestication with subsequent expansion of pandemic clones on rice and weeds of rice. Genetics 170, 613-630 (2005).

17. Stukenbrock, E. H., Banke, S., Javan-Nikkhah, M. \& McDonald, B. A. Origin and domestication of the fungal wheat pathogen Mycosphaerella graminicola via sympatric speciation. Mol. Biol. Evol. 24, 398-411 (2007).

18. Gladieux, P. et al. On the Origin and Spread of the Scab Disease of Apple: Out of Central Asia. PLOS ONE 3, e1455 (2008).

19. Munkacsi, A. B., Stoxen, S. \& May, G. Ustilago maydis populations tracked maize through domestication and cultivation in the Americas. Proc. Biol. Sci. 275, 1037-1046 (2008).

20. Ali, S. et al. Origin, Migration routes and worldwide population genetic structure of the wheat yellow rust pathogen Puccinia striiformis f.sp. tritici. PLOS Pathogens 10, e1003903 (2014).

21. Goss, E. M. et al. The Irish potato famine pathogen Phytophthora infestans originated in central Mexico rather than the Andes. PNAS 111, 8791-8796 (2014).

22. Nelson, S. C. \& Abad, Z. G. Phytophthora morindae, a new species causing black flag disease on noni (Morinda citrifolia L) in Hawaii. Mycologia 102, 122-134 (2010).

23. Érsek, T. \& Ribeiro, O. Mini Review Article: An annotated list of new Phytophthora species described post 1996. Acta Phytopathologica et Entomologica Hungarica 45, 251266 (2010).

24. Hansen, E. M., Reeser, P. W. \& Sutton, W. Phytophthora beyond agriculture. Annual Review of Phytopathology 50, 359-378 (2012).

25. Kroon, L. P. N. M., Brouwer, H., de Cock, A. W. A. M. \& Govers, F. The genus Phytophthora anno 2012. Phytopathology 102, 348-364 (2012).

26. Erwin, D. C. \& Ribeiro, O. K. Phytophthora diseases worldwide. American Phytopathological Society (APS Press), St. Paul, MN, USA (1996).

27. Fry, W. E. et al. Five reasons to consider Phytophthora infestans a reemerging pathogen. Phytopathology 105, 966-981 (2015).

541 28. Grünwald, N. J., Garbelotto, M., Goss, E. M., Heungens, K. \& Prospero, S. Emergence of the sudden oak death pathogen Phytophthora ramorum. Trends Microbiol. 20, 131-138 
(2012).

29. John T. Kliejunas. Sudden oak death and Phytophthora ramorum: a summary of the literature. Gen. Tech. Rep. PSW-GTR-234. U.S. Department of Agriculture, Forest Service, Pacific Southwest Research Station, Albany, CA (2010).

30. Mchau, G. R. A. \& Coffey, M. D. Isozyme diversity in Phytophthora palmivora: Evidence for a southeast Asian centre of origin. Mycological Research 98, 1035-1043 (1994).

31. Lamour, K. Phytophthora: A Global Perspective. (CABI, 2013).

32. Torres, G. A. et al. Bud Rot Caused by Phytophthora palmivora: A Destructive emerging disease of oil palm. Phytopathology 106, 320-329 (2016).

33. Ali, S. S. et al. Phytophthora megakarya and P. palmivora, closely related causal agents of cacao black pod rot, underwent increases in genome sizes and gene numbers by different mechanisms. Genome Biol Evol (2017). doi:10.1093/gbe/evx021

34. Zentmyer, G. A. Origin and distribution of four species of Phytophthora. Transactions of the British Mycological Society 91, 367-378 (1988).

35. Oudemans, P. \& Coffey, M. D. A revised systematics of twelve papillate Phytophthora species based on isozyme analysis. Mycological Research 95, 1025-1046 (1991).

36. Pritchard, J. K., Stephens, M. \& Donnelly, P. Inference of population structure using multilocus genotype data. Genetics 155, 945-959 (2000).

37. Drummond, A. J. \& Rambaut, A. BEAST: Bayesian evolutionary analysis by sampling trees. BMC Evol. Biol. 7, 214 (2007).

38. Cornuet, J.-M. et al. Inferring population history with DIY ABC: a user-friendly approach to approximate Bayesian computation. Bioinformatics 24, 2713-2719 (2008).

39. Beaumont, M. A. Approximate Bayesian computation in evolution and ecology. Annual Review of Ecology, Evolution, and Systematics 41, 379-406 (2010).

40. Beerli, P. \& Palczewski, M. Unified framework to evaluate panmixia and migration direction among multiple sampling locations. Genetics 185, 313-326 (2010).

41. Jombart, T., Devillard, S. \& Balloux, F. Discriminant analysis of principal components: A new method for the analysis of genetically structured populations. BMC Genetics 11, 94 (2010).

42. Kamvar, Z. N., Tabima, J. F. \& Grünwald, N. J. Poppr: An R package for genetic analysis of populations with clonal, partially clonal, and/or sexual reproduction. PeerJ 2, e281 (2014).

43. Kamvar, Z. N., Brooks, J. C. \& Grünwald, N. J. Novel R tools for analysis of genome-wide population genetic data with emphasis on clonality. Front Genet 6, (2015).

44. De Maio, N., Wu, C.-H., O’Reilly, K. M. \& Wilson, D. New Routes to Phylogeography: A Bayesian structured coalescent approximation. PLOS Genetics 11, e1005421 (2015).

45. Lemey, P., Rambaut, A., Drummond, A. J. \& Suchard, M. A. Bayesian phylogeography finds its roots. PLOS Computational Biology 5, e1000520 (2009).

46. Drummond, A. J., Suchard, M. A., Xie, D. \& Rambaut, A. Bayesian phylogenetics with 
BEAUti and the BEAST 1.7. Mol. Biol. Evol. 29, 1969-1973 (2012).

47. Gunn, B. F., Baudouin, L. \& Olsen, K. M. Independent origins of cultivated coconut (Cocos nucifera L.) in the Old World tropics. PLOS ONE 6, e21143 (2011).

48. Fay, J. C. \& Wu, C. I. A human population bottleneck can account for the discordance between patterns of mitochondrial versus nuclear DNA variation. Mol. Biol. Evol. 16, 1003-1005 (1999).

49. Adler, N. E. et al. Genetic Diversity of Phytophthora infestans sensu lato in Ecuador provides new insight into the origin of this important plant pathogen. Phytopathology 94, 154-162 (2004).

50. Goss, E. M. Genome-enabled analysis of plant-pathogen migration. Annual Review of Phytopathology 53, 121-135 (2015).

51. Buggs, R. J. A., Soltis, P. S. \& Soltis, D. E. Does hybridization between divergent progenitors drive whole-genome duplication? Mol. Ecol. 18, 3334-3339 (2009).

52. Motamayor, J. C. et al. Cacao domestication I: the origin of the cacao cultivated by the Mayas. Heredity 89, 380-386 (2002).

53. Verheye, W. Growth and production of rubber. Land Use, Land Cover and Soil Sciences. Encyclopedia of life support systems (EOLSS), UNESCO-EOLSS Publishers, Oxford, UK (2010).

54. Hine, R. B. Pathogenicity of Phytophthora palmivora in the Orchidaceae. Plant Dis. Rep. 46, 643-645 (1962).

55. Baudouin, L. \& Lebrun, P. Coconut (Cocos nucifera L.) DNA studies support the hypothesis of an ancient Austronesian migration from Southeast Asia to America. Genet Resour Crop Evol 56, 257-262 (2009).

56. Roullier, C., Benoit, L., McKey, D. B. \& Lebot, V. Historical collections reveal patterns of diffusion of sweet potato in Oceania obscured by modern plant movements and recombination. PNAS 110, 2205-2210 (2013).

57. Wood, G. R. A history of early cocoa introductions. Cocoa Growers' Bull. 44, 7-12 (1991).

58. Gin, O. K. Southeast Asia : A Historical Encyclopedia, From Angkor Wat to East Timor. ABC-CLIO, Inc., Santa Barbara, CA, USA (2004).

59. Hallucinogenic Plants: Richard Evans Schultes, Elmer W. Smith: 9780307243621: Amazon.com: Books. Available at: https://www.amazon.com/Hallucinogenic-PlantsRichard-Evans-Schultes/dp/0307243621. (Accessed: 16th March 2019)

60. Frank Z. The International Natural Rubber Market 1870-1930. Economic History Association (2010).

61. Robert, S., Ravigne, V., Zapater, M.-F., Abadie, C. \& Carlier, J. Contrasting introduction scenarios among continents in the worldwide invasion of the banana fungal pathogen Mycosphaerella fijiensis. Mol. Ecol. 21, 1098-1114 (2012).

62. Saleh, D., Milazzo, J., Adreit, H., Fournier, E. \& Tharreau, D. South-East Asia is the center of origin, diversity and dispersion of the rice blast fungus, Magnaporthe oryzae. New Phytol. 201, 1440-1456 (2014). 
63. Grünwald, N. J. \& Flier, W. G. The biology of Phytophthora infestans at its center of origin. Annu Rev Phytopathol 43, 171-190 (2005).

64. Gómez-Alpizar, L., Carbone, I. \& Ristaino, J. B. An Andean origin of Phytophthora infestans inferred from mitochondrial and nuclear gene genealogies. PNAS 104, 3306-3311 (2007).

65. Wang, J. et al. High levels of diversity and population structure in the potato late blight pathogen at the Mexico centre of origin. Mol. Ecol. 26, 1091-1107 (2017).

66. Bebber, D. P., Holmes, T. \& Gurr, S. J. The global spread of crop pests and pathogens. Global Ecology and Biogeography 23, 1398-1407 (2014).

67. Martin, F. N. Mitochondrial haplotype determination in the oomycete plant pathogen Phytophthora ramorum. Curr Genet 54, 23-34 (2008).

68. Blair, J. E., Coffey, M. D., Park, S.-Y., Geiser, D. M. \& Kang, S. A multi-locus phylogeny for Phytophthora utilizing markers derived from complete genome sequences. Fungal Genet. Biol. 45, 266-277 (2008).

69. Goss, E. M., Carbone, I. \& Grünwald, N. J. Ancient isolation and independent evolution of the three clonal lineages of the exotic sudden oak death pathogen Phytophthora ramorum. Mol. Ecol. 18, 1161-1174 (2009).

70. Stephens, M., Smith, N. J. \& Donnelly, P. A new statistical method for haplotype reconstruction from population data. Am. J. Hum. Genet. 68, 978-989 (2001).

71. Li, N. \& Stephens, M. Modeling linkage disequilibrium and identifying recombination hotspots using single-nucleotide polymorphism data. Genetics 165, 2213-2233 (2003).

72. Kearse, M. et al. Geneious Basic: An integrated and extendable desktop software platform for the organization and analysis of sequence data. Bioinformatics 28, 1647-1649 (2012).

73. Nei, M. Molecular Evolutionary Genetics. Columbia University Press, New York, NY, USA (1987).

74. Nei, M. \& Miller, J. C. A simple method for estimating average number of nucleotide substitutions within and between populations from restriction data. Genetics 125, 873-879 (1990).

75. Watterson, G. A. On the number of segregating sites in genetical models without recombination. Theor Popul Biol 7, 256-276 (1975).

76. Kimura, M. The Neutral Theory of Molecular Evolution. Cambridge University Press, Cambridge, UK (1983). doi:10.1017/CBO9780511623486

77. Tajima, F. Evolutionary relationship of DNA sequences in finite populations. Genetics 105, 437-460 (1983).

78. Tajima, F. Statistical method for testing the neutral mutation hypothesis by DNA polymorphism. Genetics 123, 585-595 (1989).

79. Tajima, F. Measurement of DNA polymorphism. Takahata N, Clark AG (eds), Mechanisms of Molecular Evolution., Sinauer Associates Inc, Sunderland, MA, USA, 37-59 (1993).

80. Ewens, W. J. The sampling theory of selectively neutral alleles. Theoretical Population 
Biology 3, 87-112 (1972).

81. Strobeck, C. Average number of nucleotide differences in a sample from a single subpopulation: a test for population subdivision. Genetics 117, 149-153 (1987).

82. Fu, Y. X. \& Li, W. H. Statistical tests of neutrality of mutations. Genetics 133, 693-709 (1993).

83. Simonsen, K. L., Churchill, G. A. \& Aquadro, C. F. Properties of statistical tests of neutrality for DNA polymorphism data. Genetics 141, 413-429 (1995).

84. Fu, Y. X. Statistical properties of segregating sites. Theor Popul Biol 48, 172-197 (1995).

85. Fu, Y. X. Statistical tests of neutrality of mutations against population growth, hitchhiking and background selection. Genetics 147, 915-925 (1997).

86. Hudson, R. R. \& Kaplan, N. L. Statistical properties of the number of recombination events in the history of a sample of DNA sequences. Genetics 111, 147-164 (1985).

87. Librado, P. \& Rozas, J. DnaSP v5: A software for comprehensive analysis of DNA polymorphism data. Bioinformatics 25, 1451-1452 (2009).

88. Excoffier, L. \& Lischer, H. E. L. Arlequin suite ver 3.5: A new series of programs to perform population genetics analyses under Linux and Windows. Mol Ecol Resour 10, 564567 (2010).

89. Falush, D., Stephens, M. \& Pritchard, J. K. Inference of population structure using multilocus genotype data: Linked loci and correlated allele frequencies. Genetics 164, 1567-1587 (2003).

90. Halkett, F., Simon, J.-C. \& Balloux, F. Tackling the population genetics of clonal and

91. Kaeuffer, R., Réale, D., Coltman, D. W. \& Pontier, D. Detecting population structure using STRUCTURE software: effect of background linkage disequilibrium. Heredity (Edinb) 99 , 374-380 (2007).

92. Evanno, G., Regnaut, S. \& Goudet, J. Detecting the number of clusters of individuals using the software STRUCTURE: a simulation study. Mol. Ecol. 14, 2611-2620 (2005).

93. Earl, D. A. \& vonHoldt, B. M. STRUCTURE HARVESTER: a website and program for visualizing STRUCTURE output and implementing the Evanno method. Conservation Genet Resour 4, 359-361 (2012).

94. Jakobsson, M. \& Rosenberg, N. A. CLUMPP: a cluster matching and permutation program for dealing with label switching and multimodality in analysis of population structure.

694 95. Team RC. R: A language and environment for statistical computing. (2013).

695 96. Darriba, D., Taboada, G. L., Doallo, R. \& Posada, D. jModelTest 2: more models, new heuristics and parallel computing. Nat. Methods 9, 772 (2012). http://beast.bio.ed.ac.uk/Tracer (2014).

98. Heled, J. \& Bouckaert, R. R. Looking for trees in the forest: Summary tree from posterior 
samples. BMC Evolutionary Biology 13, 221 (2013).

99. Sánchez-Ramírez, S. rBt: Handy functions for dealing with BEAST trees in R. GitHub repository: https://github.com/santiagosnchez/rBt (2018).

100. Bouckaert, R. et al. BEAST 2: A software platform for Bayesian evolutionary analysis. PLOS Computational Biology 10, e1003537 (2014).

101. Vaughan, T. G. IcyTree: rapid browser-based visualization for phylogenetic trees and networks. Bioinformatics 33, 2392-2394 (2017).

102. Marin-Burgos, V., Clancy, J. S. Understanding the expansion of energy crops beyond the global biofuel boom: evidence from oil palm expansion in Colombia. Energ. Sustain. Soc. 7, 21 (2017). https://doi.org/10.1186/s13705-017-0123-2

103. Drenth, A. \& Sendall, B. Economic impact of Phytophthora diseases in Southeast Asia. Drenth, A. \& Guest, D.I., eds. Diversity and management of Phytophthora in Southeast Asia. Australian Centre for International Agricultural Research (ACIAR), Canberra, Australia, ACIAR Monograph No. 114, 10-28 (2004).

716 We thank Jordan McLendon for assistance in the laboratory, Sebastian Schornack and Sophien

717 Kamoun for sharing P. palmivora genome data for primer design, and Santiago Sánchez-

718 Ramírez for assistance with $r B t$. This work was supported by the University of Florida

719 Department of Plant Pathology and Emerging Pathogens Institute.

721 Author contributions

722 JW and EMG designed the experiments. MDC contributed the DNA samples of Phytophthora

723 palmivora from the World Oomycete Genetic Resource Collection. JW performed the data

724 analyses with contributions from EMG and NDM. JW and EMG wrote the manuscript with

725 contribution from NDM and MDC. All authors reviewed and approved the manuscript.

\section{Competing interests}

728 The authors declare no competing financial interests. 


\section{TABLES}

Table 1. Nucleotide variation by locus and geographic region. Statistics given are number of individuals (Nind), number of sequences (Nseq), number of haplotypes (Nhap), segregating sites (S), average pairwise nucleotide diversity $(\pi)$, Watterson's theta $(\theta \mathrm{w})$,

Tajima's D, number of mutations, number of singleton mutations, Fu and Li's D* and F*, and minimum number of recombination events $(\mathrm{Rm})$.

\begin{tabular}{|c|c|c|c|c|c|c|c|c|c|c|c|c|c|}
\hline Locus & $\begin{array}{l}\text { Geographic } \\
\text { region }\end{array}$ & Nind & Nseq & Nhap & $\mathrm{S}$ & $\Pi$ & $\theta \mathrm{w}$ & $\begin{array}{l}\text { Tajima's } \\
\text { D }\end{array}$ & $\begin{array}{l}\text { Number } \\
\text { of } \\
\text { mutations }\end{array}$ & $\begin{array}{l}\text { Number } \\
\text { of } \\
\text { singleton } \\
\text { mutations }\end{array}$ & $\begin{array}{l}\text { Fu \& Li's } \\
\text { D* }\end{array}$ & $\begin{array}{l}\text { Fu \& Li’s } \\
F^{*}\end{array}$ & $\mathrm{Rm}$ \\
\hline \multirow[t]{3}{*}{$\beta$-tubulin } & Africa & 6 & 12 & 4 & 23 & 0.0076 & 7.6 & 1.53 & 23 & 6 & 0.44 & 0.82 & 1 \\
\hline & America & 18 & 36 & 7 & 30 & 0.0072 & 7.2 & 1.20 & 30 & 10 & -0.61 & $<-0.01$ & 6 \\
\hline & Asia-Pacific & 66 & 132 & 45 & 55 & 0.0088 & 10.1 & 0.56 & 55 & 11 & -0.19 & 0.15 & 14 \\
\hline \multirow[t]{3}{*}{ pelota } & Africa & 6 & 12 & 6 & 14 & 0.0097 & 4.6 & 0.96 & 15 & 5 & 0.12 & 0.38 & 0 \\
\hline & America & 18 & 36 & 8 & 20 & 0.0091 & 4.8 & 0.63 & 20 & 8 & -1.01 & -0.56 & 3 \\
\hline & Asia-Pacific & 68 & 136 & 38 & 36 & 0.0108 & 6.6 & -0.06 & 38 & 13 & -1.82 & -1.31 & 8 \\
\hline \multirow[t]{3}{*}{$\operatorname{trpl}$} & Africa & 5 & 10 & 5 & 9 & 0.0053 & 3.2 & 1.42 & 10 & 2 & 0.71 & 1.00 & 0 \\
\hline & America & 16 & 32 & 6 & 10 & 0.0048 & 2.5 & 2.19 & 10 & 2 & 0.31 & 1.05 & 2 \\
\hline & Asia-Pacific & 69 & 138 & 24 & 35 & 0.0045 & 6.4 & -1.24 & 37 & 10 & -1.00 & -1.32 & 6 \\
\hline \multirow{3}{*}{$\begin{array}{l}\text { coxII and } \\
\text { spacer }\end{array}$} & Africa & 5 & 5 & 2 & 10 & 0.0068 & 4.8 & -1.19 & 10 & 10 & -1.19 & -1.26 & 0 \\
\hline & America & 17 & 17 & 4 & 7 & 0.0018 & 2.1 & -2.00 & 8 & 7 & -2.41 & -2.65 & 0 \\
\hline & Asia-Pacific & 62 & 62 & 28 & 24 & 0.0083 & 5.1 & -0.25 & 25 & 3 & 0.80 & 0.50 & 7 \\
\hline
\end{tabular}

Bold: $P<0.05$. 
Table 2. Analysis of molecular variance among three major geographic regions and two major hosts.

\begin{tabular}{llll}
\hline Loci & Source of variation & Percentage of variation & FST $^{*}$ \\
\hline$\beta$-tubulin & Among regions & 7.77 & 0.08 \\
pelota & & 10.01 & 0.10 \\
trpl & 11.54 & 0.12 \\
coxII and spacer & Among hosts & 22.64 & 0.08 \\
\hline$\beta$-tubulin & & 27.38 & 0.23 \\
pelota & & 23.97 & 0.27 \\
trpl & & 11.90 & 0.24 \\
coxII and spacer & & 0.12
\end{tabular}

*All values of FST were statistically significant with $P<0.05$. 
Table 3. Estimates of migration rates between demes defined by hosts and geographical regions.

a. Three-deme model*

\begin{tabular}{lllll}
\hline \multirow{2}{*}{$\begin{array}{l}\text { Source deme } \\
\text { Cacao-America }\end{array}$} & Root state probability & Cacao-America & Cacao-AsiaPacific & Coconut-Asia \\
\hline Cacao-AsiaPacific & $0.93-0.94$ & - & $\mathbf{0 . 4 4 7}$ & 0.108 \\
& & & {$[0.158-\mathbf{0 . 7 8 5}]$} & {$[0-0.271]$} \\
Coconut-Asia & $<0.1$ & 0.069 & - & $\mathbf{0 . 3 0 6}$ \\
& & {$[0-0.188]$} & 0.043 & {$[\mathbf{0 . 0 7 6 - 0 . 5 7 5 ]}$} \\
& & 0.031 & {$[0-0.130]$} & - \\
\hline
\end{tabular}

* P.palmivora isolates from coconuts in America were not present in our sample

Bold: lower boundary of $95 \%$ HPD $>0.001$

b. Four-deme model, including an unsampled deme

\begin{tabular}{llllll}
\hline & & & \multicolumn{3}{c}{ Mean estimated immigration rate [95\% HPD]: } \\
Source deme & Root state probability & Cacao-America & Cacao-AsiaPacific & Coconut-Asia & Unsampled \\
\hline Cacao-America & $0.63-0.68$ & - & $\mathbf{0 . 4 7 6}$ & 0.11 & 0.069 \\
& & & {$[\mathbf{0 . 1 7 1 - 0 . 8 2 7}]$} & {$[0-0.273]$} & {$[0-0.212]$} \\
Cacao-AsiaPacific & $<0.1$ & 0.063 & - & $\mathbf{0 . 3 1 4}$ & 0.082 \\
& & {$[0-0.180]$} & & {$[\mathbf{0 . 0 5 3 - 0 . 6 0 4}]$} & {$[0-0.248]$} \\
Coconut-Asia & $<0.1$ & 0.031 & 0.048 & - & 0.085 \\
& & {$[0-0.092]$} & {$[0-0.146]$} & & {$[0-0.252]$} \\
Unsampled & $0.27-0.31$ & 0.076 & 0.113 & 0.272 & - \\
& & {$[0-0.192]$} & {$[0-0.321]$} & {$[0-0.606]$} &
\end{tabular}

Bold: lower boundary of 95\% HPD > 0.001 
c. Four-deme model, including a putative Others-AsiaPacific deme

Mean estimated immigration rate $[95 \% \mathrm{HPD}]$ :

\begin{tabular}{|c|c|c|c|c|c|}
\hline Source deme & Root state probability & Cacao-America & Cacao-AsiaPacific & Coconut-Asia & Others-AsiaPacific \\
\hline Cacao-America & $0.95-0.97$ & - & $\begin{array}{l}0.403 \\
{[0.149-0.698]}\end{array}$ & $\begin{array}{l}0.104 \\
{[0-0.228]}\end{array}$ & $\begin{array}{l}0.222 \\
{[0.034-0.433]}\end{array}$ \\
\hline Cacao-AsiaPacific & $<0.1$ & $\begin{array}{l}0.037 \\
{[0-0.110]}\end{array}$ & - & $\begin{array}{l}0.195 \\
{[0.004-0.395]}\end{array}$ & $\begin{array}{l}0.316 \\
{[0.004-0.599]}\end{array}$ \\
\hline Coconut-Asia & $<0.1$ & $\begin{array}{l}0.018 \\
{[0-0.054]}\end{array}$ & $\begin{array}{l}0.047 \\
{[0-0.139]}\end{array}$ & - & $\begin{array}{l}0.359 \\
{[0.148-0.599]}\end{array}$ \\
\hline Others-AsiaPacific & $<0.1$ & $\begin{array}{l}0.028 \\
{[0-0.081]}\end{array}$ & $\begin{array}{l}0.050 \\
{[0-0.153]}\end{array}$ & $\begin{array}{l}0.052 \\
{[0-0.152]}\end{array}$ & - \\
\hline
\end{tabular}

Bold: lower boundary of 95\% HPD > 0.001 